\title{
An international genome-wide meta-analysis of primary biliary cholangitis: Novel risk loci and candidate drugs
}

\section{Graphical abstract}

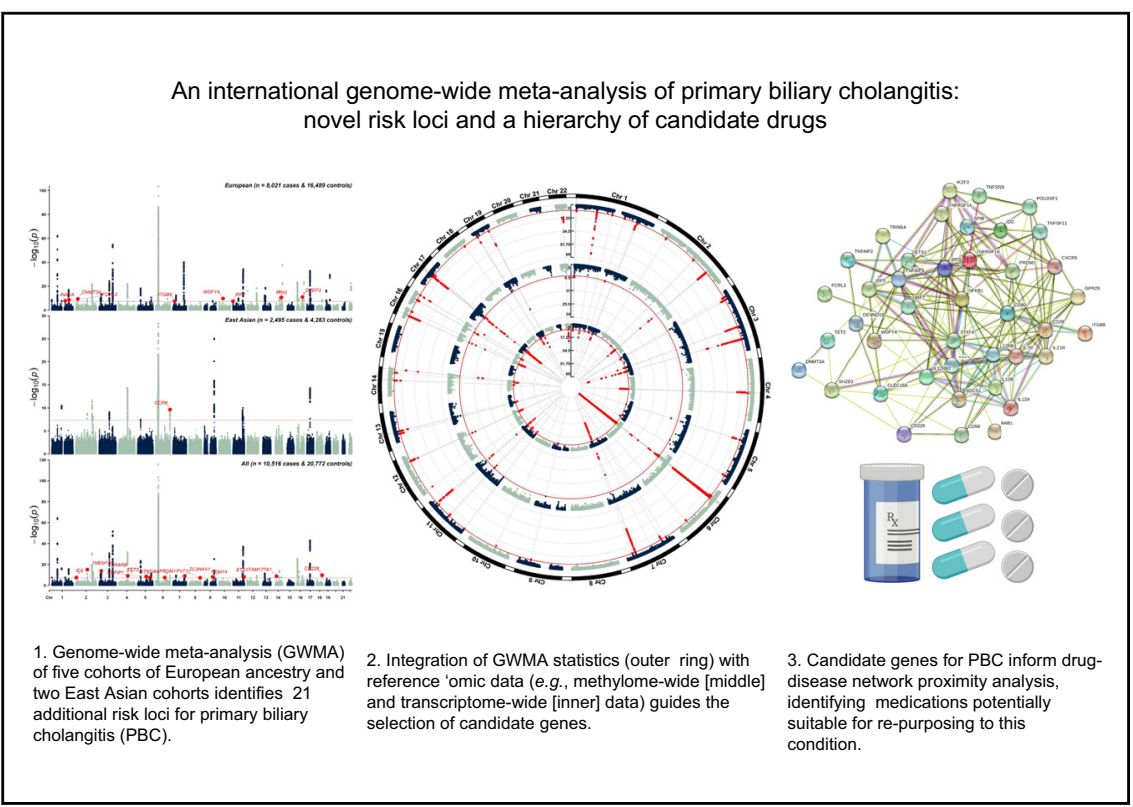

\section{Highlights}

- Trans-ethnic genome-wide meta-analysis (GWMA) of susceptibility to primary biliary cholangitis (PBC).

- Five cohorts of European ancestry and two East Asian cohorts ( $\mathrm{n}=$ 10,516 cases and 20,772 controls).

- Identification of 21 additional risk loci for PBC.

- Preliminary evidence that the genetic architecture of PBC is broadly shared across European and East Asian populations.

- Identification (using in silico drug efficacy screening) of medications potentially suitable for re-purposing to PBC.

\section{Authors}

Heather J. Cordell, James J. Fryett, Kazuko Ueno, ..., Xiong Ma, Minoru Nakamura, George F. Mells

\section{Correspondence}

gfm26@cam.ac.uk (G.F. Mells).

\section{Lay summary}

Primary biliary cholangitis (PBC) is a chronic liver disease that eventually leads to cirrhosis. In this study, we analysed genetic information from 10,516 people with PBC and 20,772 healthy individuals recruited in Canada, China, Italy, Japan, the UK, or the USA. We identified several genetic regions associated with PBC. Each of these regions contains several genes. For each region, we used diverse sources of evidence to help us choose the gene most likely to be involved in causing PBC. We used these 'candidate genes' to help us identify medications that are currently used for treatment of other conditions, which might also be useful for treatment of PBC. 


\title{
An international genome-wide meta-analysis of primary biliary cholangitis: Novel risk loci and candidate drugs
}

\author{
Heather J. Cordell ${ }^{1}$, James J. Fryett ${ }^{1}$, Kazuko Ueno ${ }^{2}$, Rebecca Darlay ${ }^{1}$, Yoshihiro Aiba ${ }^{3}$, \\ Yuki Hitomi $^{4}$, Minae Kawashima ${ }^{4}$, Nao Nishida ${ }^{4}$, Seik-Soon Khor ${ }^{2}$, Olivier Gervais ${ }^{5,6}$, \\ Yosuke Kawai $^{2}$, Masao Nagasaki ${ }^{5,6}$, Katsushi Tokunaga ${ }^{2}$, Ruqi Tang ${ }^{7}$, Yongyong Shi ${ }^{8,9}$, \\ Zhiqiang Li ${ }^{8,9}$, Brian D. Juran ${ }^{10}$, Elizabeth J. Atkinson ${ }^{11}$, Alessio Gerussi ${ }^{12,13}$, Marco Carbone ${ }^{12,13}$, \\ Rosanna Asselta ${ }^{14,15}$, Angela Cheung ${ }^{10}$, Mariza de Andrade ${ }^{11}$, Aris Baras ${ }^{16}$, Julie Horowitz ${ }^{16}$, \\ Manuel A.R. Ferreira ${ }^{16}$, Dylan Sun ${ }^{16}$, David E. Jones ${ }^{17}$, Steven Flack ${ }^{18}$, Ann Spicer ${ }^{18}$, \\ Victoria L. Mulcahy ${ }^{18}$, Jinyoung Byan ${ }^{19}$, Younghun Han ${ }^{19}$, Richard N. Sandford ${ }^{18}$, \\ Konstantinos N. Lazaridis ${ }^{10}$, Christopher I. Amos $^{19}$, Gideon M. Hirschfield ${ }^{20}$, Michael F. Seldin ${ }^{21}$, \\ Pietro Invernizzi ${ }^{12,13}$, Katherine A. Siminovitch ${ }^{22,23}$, Xiong Ma7, Minoru Nakamura ${ }^{3,24}$, \\ George F. Mells ${ }^{18, *}$, for the PBC Consortia, Canadian PBC Consortium, Chinese PBC Consortium, \\ Italian PBC Study Group, Japan-PBC-GWAS Consortium, US PBC Consortium, UK-PBC

\section{Consortium}

\begin{abstract}
${ }^{1}$ Population Health Sciences Institute, Faculty of Medical Sciences, Newcastle University, Newcastle upon Tyne, United Kingdom; ${ }^{2}$ Genome Medical Science Project, National Center for Global Health and Medicine (NCGM), Tokyo, Japan; ${ }^{3}$ Clinical Research Center, National Hospital Organization, Nagasaki Medical Center, Omura, Japan; ${ }^{4}$ Department of Human Genetics, Graduate School of Medicine, The University of Tokyo, Tokyo, Japan; ${ }^{5}$ Human Biosciences Unit for the Top Global Course Center for the Promotion of Interdisciplinary Education and Research, Kyoto University, Kyoto, Japan; ${ }^{6}$ Center for Genomic Medicine, Graduate School of Medicine, Kyoto University, Kyoto, Japan; ${ }^{7}$ Division of Gastroenterology and Hepatology, Key Laboratory of Gastroenterology and Hepatology, Ministry of Health, State Key Laboratory for Oncogenes and Related Genes, Renji Hospital, School of Medicine, Shanghai JiaoTong University, Shanghai Institute of Digestive Disease, Shanghai, China; ${ }^{8}$ Bio-X Institutes, Key Laboratory for the Genetics of Developmental and Neuropsychiatric Disorders (Ministry of Education), Collaborative Innovation Center for Brain Science, Shanghai Jiao Tong University, Shanghai, China; ${ }^{9}$ Affiliated Hospital of Qingdao University and Biomedical Sciences Institute of Qingdao University (Qingdao Branch of SJTU Bio-X Institutes), Qingdao University, Qingdao, China;

${ }^{10}$ Division of Gastroenterology and Hepatology, Mayo Clinic, Rochester, Minnesota, United States; ${ }^{11}$ Division of Biomedical Statistics and Informatics, Mayo Clinic, Rochester, Minnesota, United States; ${ }^{12}$ Division of Gastroenterology and Center for Autoimmune Liver Diseases, Department of Medicine and Surgery, University of Milano-Bicocca, Monza, Italy; ${ }^{13}$ European Reference Network on Hepatological Diseases (ERN RARE-LIVER), San Gerardo Hospital, Monza, Italy; ${ }^{14}$ Department of Biomedical Sciences, Humanitas University, Pieve Emanuele, Milan Italy; ${ }^{15}$ Humanitas Clinical and Research Center, IRCCS, Rozzano, Milan, Italy; ${ }^{16}$ Regeneron Genetics Center, Tarrytown, New York, United States; ${ }^{17}$ Translational and Clinical Research Institute, Faculty of Medical Sciences, Newcastle University, Newcastle upon Tyne, United Kingdom; ${ }^{18}$ Academic Department of Medical Genetics, University of Cambridge, Cambridge, United Kingdom; ${ }^{19}$ Institute for Clinical and Translational Research, Baylor College of Medicine, Houston, Texas, United States; ${ }^{20}$ Toronto Centre for Liver Disease, Division of Gastroenterology and Hepatology, University of Toronto, Toronto, Ontario, Canada; ${ }^{21}$ University of California, Davis, California, United States;

${ }^{22}$ Departments of Medicine, Immunology and Medical Sciences, University of Toronto, Toronto, Ontario, Canada; ${ }^{23}$ Mount Sinai Hospital, Lunenfeld-Tanenbaum Research Institute and Toronto General Research Institute, Toronto, Ontario, Canada; ${ }^{24}$ Department of Hepatology, Nagasaki Graduate School of Biomedical Sciences, Japan
\end{abstract}

\begin{abstract}
Backgrounds \& Aims: Primary biliary cholangitis (PBC) is a chronic liver disease in which autoimmune destruction of the small intrahepatic bile ducts eventually leads to cirrhosis. Many patients have inadequate response to licensed medications, motivating the search for novel therapies. Previous genomewide association studies (GWAS) and meta-analyses (GWMA) of $\mathrm{PBC}$ have identified numerous risk loci for this condition,

Keywords: UK-PBC; ERN RARE-LIVER; ALSPAC; Genomic co-localization; Networkbased in silico drug efficacy screening.

Received 25 August 2020; received in revised form 11 March 2021; accepted 7 April 2021; available online 23 May 2021

* Corresponding author. Address: Academic Department of Medical Genetics, University of Cambridge, Box 238, Addenbrooke's Hospital, Hills Road, Cambridge CB2 0QQ United Kingdom.

E-mail address: gfm26@cam.ac.uk (G.F. Mells).

https://doi.org/10.1016/j.jhep.2021.04.055
\end{abstract}

providing insight into its aetiology. We undertook the largest GWMA of PBC to date, aiming to identify additional risk loci and prioritise candidate genes for in silico drug efficacy screening. Methods: We combined new and existing genotype data for 10,516 cases and 20,772 controls from 5 European and 2 East Asian cohorts.

Results: We identified 56 genome-wide significant loci (20 novel) including 46 in European, 13 in Asian, and 41 in combined cohorts; and a $57^{\text {th }}$ genome-wide significant locus (also novel) in conditional analysis of the European cohorts. Candidate genes at newly identified loci include FCRL3, INAVA, PRDM1, IRF7, CCR6, CD226, and IL12RB1, which each play key roles in immunity. Pathway analysis reiterated the likely importance of pattern recognition receptor and TNF signalling, JAK-STAT signalling, and differentiation of $T$ helper $\left(T_{H}\right) 1$ and $T_{H} 17$ cells in the 


\section{JOURNAL OF HEPATOLOGY}

pathogenesis of this disease. Drug efficacy screening identified several medications predicted to be therapeutic in PBC, some of which are well-established in the treatment of other autoimmune disorders.

Conclusions: This study has identified additional risk loci for PBC, provided a hierarchy of agents that could be trialled in this condition, and emphasised the value of genetic and genomic approaches to drug discovery in complex disorders.

Lay summary: Primary biliary cholangitis (PBC) is a chronic liver disease that eventually leads to cirrhosis. In this study, we analysed genetic information from 10,516 people with PBC and 20,772 healthy individuals recruited in Canada, China, Italy, Japan, the UK, or the USA. We identified several genetic regions associated with $\mathrm{PBC}$. Each of these regions contains several genes. For each region, we used diverse sources of evidence to help us choose the gene most likely to be involved in causing PBC. We used these 'candidate genes' to help us identify medications that are currently used for treatment of other conditions, which might also be useful for treatment of PBC.

(C) 2021 Published by Elsevier B.V. on behalf of European Association for the Study of the Liver.

\section{Introduction}

Primary biliary cholangitis ( $\mathrm{PBC}$ ) is a chronic liver disease in which autoimmune injury to the small intrahepatic bile ducts eventually leads to cirrhosis. Only 2 medications, ursodeoxycholic acid (UDCA) and obeticholic acid (OCA), are licensed for the treatment of PBC. Many patients have inadequate response to both agents, leaving them at risk of progressive liver disease. Notwithstanding recent advances, novel therapies are needed for this condition.

Delineating the genetic architecture of PBC can provide insight into its aetiology - and more specifically, identify potential drug targets. Therefore, over the past decade, our respective groups have undertaken genome-wide association studies (GWAS) of PBC in Canadian-US, ${ }^{1}$ Italian, ${ }^{2}$ British, ${ }^{3}$ Japanese, ${ }^{4}$ and Chinese ${ }^{5}$ cohorts; and in 2015, we undertook a genome-wide meta-analysis (GWMA) of the Canadian-US, Italian, and British discovery panels. ${ }^{6}$ These studies have identified genome-wide significant associations at the human leukocyte antigen (HLA) locus and 42 non-HLA loci.

Our GWMA in 2015 did not include the Japanese or Chinese discovery panels. Furthermore, since 2015, our respective groups have undertaken genome-wide genotyping of substantially expanded Canadian, Italian, UK, and US cohorts. Therefore, we present an updated GWMA of PBC that includes these expanded cohorts, as well as the Japanese and Chinese discovery panels. In this study, we aimed to: i) capitalise on the increased sample size to discover additional risk loci for PBC; ii) explore populationspecific genetic heterogeneity at known and newly identified risk loci; iii) integrate GWMA statistics with publicly available epigenetic, gene expression, and proteomic datasets to prioritise causal variants and candidate genes; and iv) use these candidate genes for in silico drug efficacy screening to identify agents potentially suitable for re-purposing to PBC.

\section{Materials and methods}

Participants and genotyping are summarised in Table 1 and detailed in the supplementary information. Written informed consent was obtained from each participant. The research conformed to the ethical guidelines of the 1975 Declaration of Helsinki.

\section{Quality control}

For the European and Japanese panels, quality control (QC) checks were performed at Newcastle University, UK, using the software package PLINK. ${ }^{7}$ Specific QC thresholds to determine outliers were based on visual inspection and varied by panel. For the European panels, we first removed variants with minor allele frequency (MAF) $<0.01$; genotype call rate $<97 \%$ ( $<95 \%$ for the 'old' Italian, WTCCC3, and 'new' US panels); or significant deviation from Hardy Weinberg Equilibrium (HWE) $\left(p<10^{-6}\right)$. We then removed samples with rates of missing data $>2 \%$ ( $>4 \%$ for the new US panel); whole-genome heterozygosity $>3.25$ standard deviations from the mean; apparent gender discrepancies (based on X-chromosomal heterozygosity $>0.2$ for men and $<0.2$ for women); estimated proportion of identity-by-descent sharing with another sample $>0.1$ (based on subsets of between 38,000 and 97,000 variants pruned for linkage disequilibrium [LD]); or that did not cluster with the CEU HapMap2 population (based on visual inspection of the first 2 principal components). For the Japanese panel, we used the dataset described in Kawashima et al. (2012), ${ }^{4}$ except for the additional removal of 4 cases and 10 controls with apparent gender discrepancies.

All samples recruited in China were processed and analysed on Chinese servers to comply with the Regulation of the People's Republic of China on the Administration of Human Genetic Resources. Thus, for the Chinese panel, QC checks were undertaken on a local server in Shanghai, China. Variants were removed with MAF $<0.5 \%$, genotype call rate $<95 \%$, or deviation from HWE in controls $p \leq 1 \times 10^{-6}$. Samples were removed with rates of missing data $\geq 5 \%$ or pairwise identity-by-state, PI_HAT $>0.25$. Population outliers were identified for exclusion using principal component analysis.

\section{Genome-wide imputation and post-imputation quality control}

For the European and Japanese panels, we used the autosomal variants and samples passing QC to carry out genome-wide imputation within each individual panel using the Michigan Imputation Server with Eagle2 phasing, ${ }^{8}$ informed by the 1000 Genomes Phase 3 reference panel. Following imputation, we discarded variants with imputation $\mathrm{R}^{2}<0.5$; non-unique alleles at the same position; or imputation call rate $<90 \%$ (based on assigning genotypes according to the most likely genotype call and setting genotypes to missing if the most likely genotype call had posterior probability <0.9). We also used the resulting common set of imputed variants to check for sample duplicates/ relationships across the European panels (based on estimated identity-by-descent sharing using 25,873 variants pruned for LD) and removed 1 person from each of the 137 identified relative pairs.

For the Chinese panel, genome-wide imputation was undertaken on a local server in Shanghai, China, using SHAPEIT ${ }^{9}$ and IMPUTE2, ${ }^{10}$ and the 1000 Genomes Phase 3 reference panel. Following imputation, we discarded variants with call rates $<95 \%$ (having set genotypes to missing if the most likely genotype call had posterior probability $<0.9$ ), MAF $<0.01$, or HWE $p<1 \times 10^{-6}$ in controls. The resulting imputation summary statistics (log odds ratios [lnORs], standard errors, and $p$ values) were submitted 
Table 1. Discovery panels included in the current study.

\begin{tabular}{|c|c|c|c|c|}
\hline Panel (Ref) & Cases & Controls & Variants* & Platform \\
\hline \multicolumn{5}{|l|}{ European panels } \\
\hline 'Old' Italian (2) & 444 & 901 & $13,113,694$ & $\begin{array}{l}\text { Illumina Human610-Quad (Cases), Illumina } 1 \mathrm{M}-\text { Duo } \\
\text { (Controls) }\end{array}$ \\
\hline WTCCC3 (3) & 1,816 & 5,155 & $12,881,032$ & $\begin{array}{l}\text { Illumina Human-660 W Quad (Cases), Illumina } 1 \mathrm{M}-\text { Duo } \\
\text { (Controls) }\end{array}$ \\
\hline 'New' Canadian-UK & 4,615 & 9,233 & $8,656,760$ & Illumina HumanCoreExome \\
\hline 'New’ Italian & 255 & 579 & $9,264,788$ & Illumina HumanCoreExome \\
\hline 'New' US & 891 & 621 & $9,964,354$ & Illumina Infinium Global Screening Array (GSA) v1 \\
\hline European combined & 8,021 & 16,489 & $5,186,747$ & - \\
\hline \multicolumn{5}{|l|}{ Asian panels } \\
\hline Japanese (4) & 1,377 & 1,495 & $7,308,269$ & Affymetrix Axiom Genome-Wide ASI 1 \\
\hline Chinese (5) & 1,118 & 2,788 & $6,934,908$ & HumanOmniZhongHua-8 \\
\hline Asian combined & 2,495 & 4,283 & $5,347,815$ & - \\
\hline All combined & 10,516 & 20,772 & $2,817,608$ & - \\
\hline
\end{tabular}

WTCCC3, Wellcome Trust Case-Control Consortium 3.

*Number of variants following pre- and post-imputation quality control.

without individual-level data to Newcastle, UK, for meta-analysis with the other panels.

\section{Statistical analysis of European and Japanese cohorts}

Within each panel, we performed association analysis of the genome-wide imputed data using logistic regression of disease phenotype on single nucleotide polymorphism (SNP) genotype (coded 0,1,2) in PLINK,' with the first 10 principal components (from a pruned set of SNPs with the HLA region removed) included as covariates to correct for population stratification. (The rationale for removing the HLA region was that inclusion of SNPs in this region would risk generating components that explain variation primarily caused by strong HLA-disease association, rather than population stratification.) For all but the new Canadian-UK panel, the resulting genomic control (GC) inflation factor $\lambda$ was modest $(<1.026)$; therefore, we carried out GC correction within each panel by multiplying the standard error (SE) of the estimated lnOR for each SNP by $\sqrt{ } \lambda$. For the new Canadian-UK panel, $\lambda$ was somewhat inflated at 1.091 ; therefore, we re-analysed the new Canadian-UK data using a logistic mixed model score test (including the first 10 principal components as covariates) as implemented in the GMMAT package, ${ }^{11}$ resulting in a slightly deflated $\lambda$ of 0.971 . The SE of the estimated lnOR for each SNP from PLINK was then (conservatively) adjusted to match that implied by the GMMAT test statistic. Specifically, we multiplied the PLINK-derived SE for each SNP by a SNP-specific factor $\gamma$, where $\gamma$ was chosen so that the resulting $\chi^{2}$ test statistic $(\operatorname{lnOR} / \gamma \mathrm{SE})^{2}$ for that SNP had a $p$ value equal to the $p$ value from GMMAT. GC correction was also performed for the Chinese summary statistics $(\lambda=1.050)$ by multiplying the SE of the estimated lnOR for each SNP by $\sqrt{ } \lambda$.

Meta-analysis of European, Asian, and combined cohorts We used the software package META ${ }^{12}$ to perform fixed-effect meta-analysis of the resulting lnORs and adjusted SEs from i) the 5 European panels; ii) the 2 Asian panels; and (3) all 7 panels, in each case restricting the analysis to variants that (following post-imputation QC) appeared within all panels. Within each meta-analysed set (European, Asian, and combined), a further GC correction was performed (to adjust for the inflation factors of 1.041, 1.033, and 1.080, seen within the European, Asian, and combined cohorts, respectively) to produce the final set of genome-wide results. Specifically, as for the individual panels above, the SE of the final lnOR for each SNP was multiplied by $\sqrt{ } \lambda$, and the test statistic and $p$ value were re-calculated accordingly. This use of "double" GC correction might be considered overly conservative, given that part of the observed inflation could be due to polygenicity. We explored this using LD score regression $(\text { LDSR })^{13}$ to compare our original results with those obtained using no GC (or GMMAT-derived) correction at all. We also compared our results from all panels combined with those obtained using trans-ethnic meta-regression analysis as implemented in the software package MR-MEGA ${ }^{14}$ (see supplementary information for details).

\section{Prioritisation of candidate causal variants and candidate genes}

We used the FINEMAP ${ }^{15}$ package and Conditional and Joint Analysis $(\mathrm{COJO})^{16}$ implemented within $\mathrm{GCTA}^{17}$ to refine and look for independent associations within genome-wide significant risk loci. We used FINEMAP to construct 'credible sets' of variants most likely to be causal in PBC. We used the ENSEMBL Variant Effect Predictor, ${ }^{18}$ FUMA (Functional Mapping and Annotation) GWAS $^{19}$ platform, and reference panels from the Avon Longitudinal Study of Parents and Children (ALSPAC, http://www.bristol. ac.uk/alspac/) $)^{20}$ and the INTERVAL study (http://www. donorhealth-btru.nihr.ac.uk/studies/interval-study/) ${ }^{21}$ for mapping and functional annotation of the first set of 'credible causal variants' at each risk locus.

Adapting the approach of Barbeira et al. (2018), ${ }^{22}$ we used the MetaXcan package; our European GWMA summary statistics; and reference panels from ALSPAC, the Genotype-Tissue Expression (GTEx) project (https://gtexportal.org/), ${ }^{23}$ and the INTERVAL study to derive genome-wide genetic prediction models of DNA methylation, gene expression, and serum protein levels in cases and controls. We used these models to correlate predicted DNA methylation, gene expression, and serum protein levels with disease status in methylome-wide, transcriptomewide, and serum proteome-wide association studies (MWAS, TWAS, and PWAS, respectively).

We used the moloc package ${ }^{24}$ to look for co-localisation of association signals from our GWMA of the European panels with those derived from mapping of methylation, expression, and protein-quantitative trait loci (mQTLs, eQTLs, pQTLs) in ALSPAC, 


\section{JOURNAL OF HEPATOLOGY}

the GTEx project, and the INTERVAL study, respectively. Finally, we used the DEPICT package ${ }^{25}$ to prioritise the most likely causal gene at risk loci based on gene function.

\section{Enrichment analysis}

We used the STRING Database ${ }^{26}$ to look for enrichment of protein-protein interactions and functional annotations amongst candidate genes; and the DAVID resource ${ }^{27}$ to look for enrichment of KEGG pathways by genes with minimum $p_{\mathrm{GWMA}}<0.01$.

\section{Network-based in silico drug efficacy screening}

We employed the approach of Guney et al. $(2016)^{28}$ in which known drug targets and candidate genes for a disease are used to estimate a drug-disease proximity measure, $z$, that quantifies the closeness (or proximity) of the drug and disease gene networks, respectively, correcting for the known biases of the interactome. For this analysis, we used the drug targets listed in DrugBank (https://www.drugbank.com/, accessed January 2021) and candidate genes for PBC prioritised as above. See the supplementary information for details.

\section{Results}

GWMA identifies 21 additional genome-wide significant risk loci for PBC

Following QC, the European panels consisted of 5,186,747 variants across 8,021 cases and 16,489 controls; Asian panels, 5,347,815 variants across 2,495 cases and 4,283 controls; and all panels combined, 2,817,608 variants across 10,516 cases and 20,772 controls (Table 1). Of note, there was substantial reduction in the number of variants in all panels combined compared to the European or Asian panels. This resulted from limited overlap of variants that passed post-imputation QC in the European compared to the Asian panels, explained by our use of different genotyping platforms across cohorts, and different LD patterns in Europeans compared to Asians.

GWMA of the European panels identified 46 loci at genomewide significance $\left(p<5 \times 10^{-8}\right)$; GWMA of the Asian panels, 13 loci at genome-wide significance; and GWMA of all panels combined, 41 loci at genome-wide significance (Fig. S1). Altogether, we identified 56 genome-wide significant risk loci in one or other meta-analysis (Table S1, Fig. S2). Using COJO, we identified an additional risk locus at 19p13.11 with genome-wide significance in conditional analysis of European panels $(p=$ $\left.4.66 \times 10^{-8}\right)$, having narrowly missed this threshold in the main, unconditional analysis $\left(p=6.55 \times 10^{-8}\right)$ (Fig. S2.57). Thus, a total of 57 genome-wide significant risk loci were identified in the current study. Of these, 21 were not identified in previous studies; and 2, 1q23.1 and 11q24.3, were previously identified at suggestive rather than genome-wide significance (Tables 2A\&B).,29

At 6 newly identified or newly confirmed risk loci, we considered evidence of association to be conclusive because: i) an unequivocal association signal was evident in both the European and Asian panels; and ii) where the lead variant at the locus was different in the European compared to the Asian panels, permutation testing confirmed the significance of a signal in the validating dataset, located in proximity to the primary signal in the index dataset ( $p_{\text {permutation }}<0.00217$, corresponding to $p<0.05$ Bonferroni-corrected for 23 tests; see supplementary information for details) (Table 2A, Table S1, Fig. S2).

At 17 newly identified or newly confirmed risk loci, we considered evidence of association to be strong but not conclusive because unequivocal association was evident in the European but not the Asian panels, or permutation testing was not significant at $p_{\text {permutation }}<0.00217$ (Table 2B, Table S1, Fig. S2). We note, however, that most of these loci achieved levels of significance in the Asian panels that were suggestive for validation, including 2 loci with suggestive permutation $p$ values $\left(4 q 24, p_{\text {permutation }}=0.0040\right.$; and $\left.5 q 21.1, p_{\text {permutation }}=0.0032\right)$.

We confirmed genome-wide significant associations at 34 of 43 previously identified risk loci for PBC - but not at 9 previously identified risk loci. Seven of these 9 loci nevertheless showed a convincing association signal, albeit at $p>5 \times 10^{-8}$ (Table S2, Fig. S3). We found no evidence of association at the 15q25.1 locus (harbouring IL16) that was discovered and validated in the Chinese GWAS by Qiu et al. (2017) ${ }^{5}$; this is explained by the absence of a signal in the Japanese and European panels. Coverage of the 19p13.2 locus was too sparse to test association.

Using FINEMAP and COJO, we found that at most risk loci, the association signal was best explained by a single variant - but at 16 loci, it was best explained by $\geq 2$ independent variants (Table S3). Notable examples include the 2q32.2 locus harbouring STAT4, with 3 independent variants; 3q25.33 (IL12A, 3 variants); 7q32.1 (IRF5, 2 variants); and 16p13.13 (CLEC16A, 2 variants) - all consistent with previous studies showing $\geq 2$ independent associations at each of these loci.

We compared our original results to those obtained without GC (or GMMAT-derived) correction. As expected, without correction, all loci previously identified as genome-wide significant reached slightly higher levels of significance, while a few loci that did not reach genome-wide significance in our original analysis, now (just) did so (Fig. S4 and Table S4). We also compared our original results for all panels combined with those obtained using trans-ethnic meta-regression analysis, implemented in MR-MEGA. Results from MR-MEGA were highly concordant with those from our original analysis (Fig. S5), also providing genome-wide significant confirmation of an independent association signal at $7 \mathrm{q} 32.1$, which exhibited significant heterogeneity in the direction of effects between the Asian and European cohorts (Table S5 and Fig. S6).

\section{PBC shows genetic correlation with other autoimmune conditions}

Recognising that most risk loci for PBC are also risk loci for other autoimmune conditions (Table S6), we used LDSR implemented via $\mathrm{LD} \mathrm{Hub}{ }^{30}$ to evaluate the genetic correlation between PBC (using summary statistics from our European panels) and complex traits with GWAS summary statistics in the LD Hub database. We found significant genetic correlation between PBC and other immune-mediated inflammatory disorders, including systemic lupus erythematosus (SLE, $\mathrm{rg}=0.54, p=2.87 \times 10^{-14}$ ), rheumatoid arthritis (RA, $\mathrm{rg}=0.26, p=3.77 \times 10^{-5}$ ), and inflammatory bowel disease (IBD, $\mathrm{rg}=0.23, p=6.97 \times 10^{-5}$ ) (Table S7). We were unable to test genetic correlation of $\mathrm{PBC}$ with autoimmune thyroid disease, Sjögren syndrome, or systemic sclerosis because GWAS summary statistics for these conditions were not available in LD Hub at the time of interrogation (19.09.2019).

\section{The genetic architecture of $\mathrm{PBC}$ is broadly shared across European and Asian populations}

To evaluate consistency between European and Asian signals, we applied permutation testing where warranted and standard meta-analysis measures of heterogeneity to the lead variants at 
Table 2. Newly identified or newly confirmed risk loci with replicated evidence of association.

\begin{tabular}{|c|c|c|c|c|c|c|c|c|c|}
\hline \multirow[b]{2}{*}{$\begin{array}{l}\text { Locus } \\
\text { Gene }\end{array}$} & \multicolumn{3}{|c|}{ Lead variant in the European panels } & \multicolumn{3}{|c|}{ Lead variant in the Asian panels } & \multicolumn{3}{|c|}{ Lead variant in the combined panels } \\
\hline & $\begin{array}{r}\text { Variant:A1/A2 } \\
\text { Chr:BP }\end{array}$ & $\begin{array}{r}p \text { value } \\
\boldsymbol{p}_{\text {perm. }}\end{array}$ & $\begin{array}{r}\text { Beta } \\
\text { SE }\end{array}$ & $\begin{array}{r}\text { Variant:A1/A2 } \\
\text { Chr:BP }\end{array}$ & $\begin{array}{r}p \text { value } \\
p_{\text {perm. }}\end{array}$ & $\begin{array}{r}\text { Beta } \\
\text { SE }\end{array}$ & $\begin{array}{r}\text { Variant:A1/A2 } \\
\text { Chr:BP }\end{array}$ & $p$ value & $\begin{array}{r}\text { Beta } \\
\text { SE }\end{array}$ \\
\hline $\begin{array}{l}\text { 2p25.1 } \\
\text { ID2 } \\
2 \mathrm{q} 21.3\end{array}$ & $\begin{array}{r}\text { rs891058:A/G } \\
2: 8,442,547 \\
\text { rs859767:G/A }\end{array}$ & $\begin{array}{r}5.39 \times 10^{-7} \\
- \\
1.54 \times 10^{-9}\end{array}$ & $\begin{array}{r}-0.12 \\
0.02 \\
-0.14\end{array}$ & $\begin{array}{r}\mathrm{rs} 3111414: \mathrm{C} / \mathrm{G} \\
2: 8,443,859 \\
\mathrm{rs} 842349: \mathrm{T} / \mathrm{G}\end{array}$ & $\begin{array}{r}1.75 \times 10^{-4} \\
0.0017 \\
1.76 \times 10^{-9}\end{array}$ & $\begin{array}{r}0.17 \\
0.04 \\
-0.24\end{array}$ & $\begin{array}{r}\text { rs13416555:G/C } \\
2: 8,441,735 \\
\text { rs859767:G/A }\end{array}$ & $\begin{array}{c}2.95 \times 10^{-8} \\
8.94 \times 10^{-16}\end{array}$ & $\begin{array}{r}-0.12 \\
0.02 \\
-0.16\end{array}$ \\
\hline TMEM163 & $2: 135,341,200$ & - & 0.02 & $2: 135,342,452$ & $<0.0001$ & 0.04 & $2: 135,341,200$ & & 0.02 \\
\hline $\begin{array}{l}\text { 6q21 } \\
\text { PRDM1 }\end{array}$ & $\begin{array}{r}\text { rs58926232:G/C } \\
6: 10,6563,612\end{array}$ & $6.75 \times 10^{-7}$ & $\begin{array}{l}0.14 \\
0.03\end{array}$ & $\begin{array}{r}\text { rs } 4134466: A / G \\
6: 106,577,368\end{array}$ & $\begin{array}{r}6.71 \times 10^{-7} \\
0.0001\end{array}$ & $\begin{array}{l}0.20 \\
0.04\end{array}$ & $\begin{array}{l}\text { rs742108:A/G } \\
6: 106,582,920\end{array}$ & $3.16 \times 10^{-8}$ & $\begin{array}{l}0.13 \\
0.02\end{array}$ \\
\hline $6 \mathrm{q} 27$ & rs3093024:A/G & $2.37 \times 10^{-6}$ & 0.10 & rs4709148:T/C & $2.18 \times 10^{-10}$ & -0.25 & rs968334:T/C & $3.98 \times 10^{-10}$ & 0.12 \\
\hline CCR6 & $6: 167,532,793$ & 0.0001 & 0.02 & $6: 167,521,676$ & - & 0.04 & $6: 167,526,096$ & & 0.02 \\
\hline $\begin{array}{l}11 \mathrm{q} 24.3^{*} \\
\text { ETS1 }\end{array}$ & $\begin{array}{r}\text { rs10893872:T/C } \\
11: 128,325,553\end{array}$ & $9.07 \times 10^{-6}$ & $\begin{array}{l}0.10 \\
0.02\end{array}$ & $\begin{array}{r}\text { rs11430718:G/GA } \\
11: 128,307,445\end{array}$ & $\begin{array}{r}1.11 \times 10^{-6} \\
<0.0001\end{array}$ & $\begin{array}{r}-0.19 \\
0.04\end{array}$ & $\begin{array}{r}\text { rs10893872:T/C } \\
11: 128,325,553\end{array}$ & $9.77 \times 10^{-9}$ & $\begin{array}{l}0.11 \\
0.02\end{array}$ \\
\hline $14 q 13.2$ & rs712315:A/T & $5.70 \times 10^{-7}$ & 0.15 & rs199892962:AT/A & $4.36 \times 10^{-6}$ & 0.20 & rs799469:G/A & $1.73 \times 10^{-9}$ & 0.15 \\
\hline FAM177A1 & $14: 35,409,701$ & - & 0.03 & $14: 35,646,404$ & 0.0020 & 0.04 & $14: 35,444,425$ & & 0.03 \\
\hline \multicolumn{10}{|l|}{ Table 2B } \\
\hline & \multicolumn{3}{|c|}{ Lead variant in the European panels } & \multicolumn{3}{|c|}{ Lead variant in the Asian panels } & \multicolumn{3}{|c|}{ Lead variant in the combined panels } \\
\hline $\begin{array}{l}\text { Locus } \\
\text { Gene }\end{array}$ & $\begin{array}{r}\text { Variant } \\
\text { Chr:BP } \\
\end{array}$ & $p$ value & $\begin{array}{r}\text { Beta } \\
S E \\
\end{array}$ & $\begin{array}{r}\text { Variant } \\
\text { Chr:BP } \\
\end{array}$ & $\begin{array}{r}p \text { value } \\
p_{\text {perm. }}\end{array}$ & $\begin{array}{r}\text { Beta } \\
S E \\
\end{array}$ & $\begin{array}{l}\text { Variant } \\
\text { Chr:BP } \\
\end{array}$ & $p$ value & $\begin{array}{r}\text { Beta } \\
S E \\
\end{array}$ \\
\hline $\begin{array}{l}1 \mathrm{q} 23.1^{*} \\
\text { FCRL3 }\end{array}$ & $\begin{array}{l}\text { rs945635:G/C } \\
1: 157,670,290\end{array}$ & $1.59 \times 10^{-8}$ & $\begin{array}{r}-0.12 \\
0.02\end{array}$ & $\begin{array}{r}\mathrm{rs} 60459521: \mathrm{G} / \mathrm{C} \\
1: 157,147,588\end{array}$ & $\begin{array}{r}1.25 \times 10^{-3} \\
\end{array}$ & $\begin{array}{r}-0.46 \\
0.14\end{array}$ & $\begin{array}{r}\text { rs11264790:T/C } \\
1: 157,636,074\end{array}$ & $2.25 \times 10^{-8}$ & $\begin{array}{r}-0.11 \\
0.02\end{array}$ \\
\hline $1 \mathrm{q} 32.1$ & rs55734382:T/C & $2.06 \times 10^{-9}$ & -0.14 & rs 117214467:C/T & $8.55 \times 10^{-3}$ & -0.33 & rs12122721:A/G & $6.95 \times 10^{-7}$ & -0.11 \\
\hline INAVA & 1:201,019,059 & & 0.02 & $1: 200,436,787$ & - & 0.13 & $1: 200,984,480$ & & 0.02 \\
\hline $\begin{array}{l}\text { 2p23.3 } \\
\text { DNMT3A }\end{array}$ & $\begin{array}{r}\mathrm{rs} 34655300: \mathrm{T} / \mathrm{C} \\
2: 25,514,333\end{array}$ & $5.23 \times 10^{-10}$ & $\begin{array}{l}0.14 \\
0.02\end{array}$ & $\begin{array}{r}\text { rs893589:A/G } \\
2: 25,259,442\end{array}$ & $\begin{array}{r}9.41 \times 10^{-4} \\
-\end{array}$ & $\begin{array}{l}0.15 \\
0.05\end{array}$ & $\begin{array}{r}\mathrm{rs} 6711622: \mathrm{A} / \mathrm{G} \\
2: 25,531,350\end{array}$ & $3.89 \times 10^{-8}$ & $\begin{array}{l}0.11 \\
0.02\end{array}$ \\
\hline $3 \mathrm{p} 24.2$ & rs6550965:A/C & $3.65 \times 10^{-14}$ & 0.16 & rs6807549:T/G & $1.37 \times 10^{-3}$ & 0.17 & rs6550965:A/C & $1.50 \times 10^{-14}$ & 0.15 \\
\hline RARB & $3: 25,383,587$ & & 0.02 & $3: 24,951,404$ & - & 0.05 & $3: 25,383,587$ & & 0.02 \\
\hline $\begin{array}{l}4 \mathrm{q} 24 \\
\text { TET2 }\end{array}$ & $\begin{array}{r}\text { rs7663401:C/T } \\
4: 106,128,954\end{array}$ & $2.76 \times 10^{-8}$ & $\begin{array}{r}-0.13 \\
0.02\end{array}$ & $\begin{array}{r}\text { rs79109654:T/C } \\
4: 106,170,514\end{array}$ & $\begin{array}{r}8.56 \times 10^{-5} \\
0.0040\end{array}$ & $\begin{array}{l}0.37 \\
0.09\end{array}$ & $\begin{array}{r}\text { rs2007403:T/C } \\
4: 106,131,210\end{array}$ & $6.19 \times 10^{-10}$ & $\begin{array}{l}0.13 \\
0.02\end{array}$ \\
\hline $5 q 21.1$ & rs141002831:T/TCA & $1.47 \times 10^{-7}$ & 0.12 & rs157181:A/C & $3.94 \times 10^{-5}$ & 0.21 & rs60643069:GA/G & $2.48 \times 10^{-9}$ & 0.13 \\
\hline ST8SIA4 & $5: 100,202,282$ & & 0.02 & $5: 100,103,288$ & 0.0032 & 0.05 & $5: 100,238,073$ & & 0.02 \\
\hline $\begin{array}{l}5 \mathrm{q} 31.3 \\
\text { NDFIP1 }\end{array}$ & $\begin{array}{r}\text { rs10062349:G/A } \\
5: 141,509,597\end{array}$ & $7.36 \times 10^{-8}$ & $\begin{array}{r}-0.12 \\
0.02\end{array}$ & $\begin{array}{r}\text { rs3761757:A/C } \\
5: 141,488,219\end{array}$ & $\begin{array}{r}7.48 \times 10^{-3} \\
-\end{array}$ & $\begin{array}{r}-0.14 \\
0.05\end{array}$ & $\begin{array}{r}\text { rs6874308:C/T } \\
5: 141,506,911\end{array}$ & $4.67 \times 10^{-8}$ & $\begin{array}{r}-0.11 \\
0.02\end{array}$ \\
\hline $7 \mathrm{p} 21.1$ & rs7805218:A/G & $4.12 \times 10^{-8}$ & 0.13 & rs77984571:C/G & $7.54 \times 10^{-3}$ & -0.14 & rs7786537:C/G & $1.12 \times 10^{-5}$ & -0.11 \\
\hline ITGB8 & 7:20,378,801 & & 0.02 & 7:20,512,650 & - & 0.05 & 7:20,427,776 & & 0.02 \\
\hline $\begin{array}{l}\text { 7q34 } \\
\text { ZC3HAV1 }\end{array}$ & $\begin{array}{r}\text { rs370193557:GAAT/G } \\
7: 138,729,543\end{array}$ & $1.89 \times 10^{-8}$ & $\begin{array}{l}0.12 \\
0.02\end{array}$ & $\begin{array}{r}\text { rs } 12056141: G / A \\
7: 138,797,730\end{array}$ & $\begin{array}{r}1.05 \times 10^{-3} \\
-\end{array}$ & $\begin{array}{l}0.18 \\
0.05\end{array}$ & $\begin{array}{r}\text { rs370193557:G/GAAT } \\
7: 138,729,543\end{array}$ & $9.37 \times 10^{-10}$ & $\begin{array}{r}-0.12 \\
0.02\end{array}$ \\
\hline $8 q 24.21$ & rs4733851:A/G & $2.18 \times 10^{-7}$ & 0.11 & rs $1902780: C / T$ & $5.51 \times 10^{-4}$ & -0.13 & rs4733851:G/A & $4.98 \times 10^{-8}$ & -0.11 \\
\hline PVT1 & $8: 129,264,420$ & & 0.02 & $8: 129,211,788$ & - & 0.04 & $8: 129,264,420$ & & 0.02 \\
\hline $\begin{array}{l}\text { 9q22.33 } \\
\text { TRIM14 }\end{array}$ & $\begin{array}{r}\text { rs } 11390003: \mathrm{GA} / \mathrm{G} \\
9: 100741912\end{array}$ & $2.56 \times 10^{-8}$ & $\begin{array}{r}-0.15 \\
0.03\end{array}$ & $\begin{array}{r}\text { rs } 10283737: G / T \\
9: 100,780,063\end{array}$ & $1.24 \times 10^{-3}$ & $\begin{array}{l}0.15 \\
0.05\end{array}$ & $\begin{array}{r}\text { rs } 112500293: \mathrm{T} / \mathrm{C} \\
9: 100,763,455\end{array}$ & $7.63 \times 10^{-9}$ & $\begin{array}{r}-0.15 \\
0.03\end{array}$ \\
\hline $10 q 11.23$ & rs7097397:A/G & $2.42 \times 10^{-10}$ & -0.14 & rs76129863:T/C & $4.83 \times 10^{-3}$ & 0.56 & rs7922169:T/G & $5.47 \times 10^{-8}$ & 0.11 \\
\hline WDFY4 & $10: 50,025,396$ & & 0.02 & $10: 50,437,561$ & - & 0.20 & $10: 50,045,456$ & & 0.02 \\
\hline $\begin{array}{l}11 \mathrm{p} 15.5 \\
\text { IRF7 }\end{array}$ & rs58523027:TAA/T & $4.00 \times 10^{-8}$ & -0.12 & rs3216:C/G & $8.17 \times 10^{-2}$ & -0.10 & rs9667500:G/A & $1.74 \times 10^{-4}$ & $\begin{array}{r}-0.08 \\
0.02\end{array}$ \\
\hline $\begin{array}{l}\text { IRF7 } \\
\text { 14q32.12 }\end{array}$ & $\begin{array}{r}11: 646,986 \\
\text { rs72699866:A/G }\end{array}$ & $2.89 \times 10^{-11}$ & $\begin{array}{r}0.02 \\
-0.20\end{array}$ & $\begin{array}{r}11: 214,421 \\
\text { rs76914265:G/C }\end{array}$ & $1.16 \times 10^{-4}$ & $\begin{array}{r}0.06 \\
-0.30\end{array}$ & $\begin{array}{r}11: 683,761 \\
\text { rs4904964:C/A }\end{array}$ & $2.45 \times 10^{-8}$ & $\begin{array}{r}0.02 \\
-0.12\end{array}$ \\
\hline RIN3 & $14: 93,114,787$ & & 0.03 & $14: 93,219,854$ & 0.0143 & 0.08 & $14: 93,099,867$ & & 0.02 \\
\hline $\begin{array}{l}16 \mathrm{q} 22.1 \\
\text { DPEP3 }\end{array}$ & $\begin{array}{r}\text { rs79577483:G/A } \\
16: 68,036,939\end{array}$ & $1.23 \times 10^{-11}$ & $\begin{array}{l}0.21 \\
0.03\end{array}$ & $\begin{array}{l}\text { rs698729:G/C } \\
16: 68,624,205\end{array}$ & $\begin{array}{r}1.90 \times 10^{-2} \\
-\end{array}$ & $\begin{array}{r}-0.12 \\
0.05\end{array}$ & $\begin{array}{r}\text { rs } 111644390: \mathrm{TC} / \mathrm{T} \\
16: 68,046,323\end{array}$ & $1.18 \times 10^{-9}$ & $\begin{array}{l}0.17 \\
0.03\end{array}$ \\
\hline $18 q 22.2$ & rs $1808094: \mathrm{T} / \mathrm{C}$ & $2.79 \times 10^{-9}$ & 0.13 & rs76486918:T/C & $2.72 \times 10^{-3}$ & -0.91 & rs1808094:T/C & $1.66 \times 10^{-10}$ & 0.12 \\
\hline CD226 & $18: 67,526,026$ & & 0.02 & $18: 67,081,620$ & - & 0.30 & $18: 67,526,026$ & & 0.02 \\
\hline
\end{tabular}

Results for the lead variant at newly identified or newly confirmed risk loci with $p<5 \times 10^{-8}$ in fixed-effect meta-analysis of the European, Asian, or combined panels. (A) Evidence of association was taken to be conclusive because: i) an unequivocal association signal at the same locus was observed in both the European and the Asian panels; and ii) where the lead variant at the locus was different in the European vs. the Asian panels, permutation testing confirmed the significance of the signal in the validating dataset at $p_{\text {perm }}<0.00217$ (see supplementary information and Table S1). (B) Evidence of association was taken to be strong but not conclusive because unequivocal association was evident in the European but not the Asian panels, or permutation testing was not significant at $p_{\text {perm }}<0.00217$. Gene: candidate gene at the risk locus (which is not necessarily the mapped gene). A1, tested allele; $\mathrm{A} 2$, alternative allele; BP, base pair position; Chr, chromosome; $p_{\text {perm, }}$, permutation $p$ value; OR, odds ratio.

${ }^{*}$ Note that 1q23.1 and 11q24.3 were previously identified at suggestive level of significance in the study by Kawashima et al. (2017). 


\section{JOURNAL OF HEPATOLOGY}

each of the 56 genome-wide significant risk loci identified or confirmed in the main, unconditional analyses (Table S1). We found concordance between risk loci operating in European and Asian populations, considering i) the much smaller sample size of the Asian panels; and ii) the interrogation of different variants in the European compared to the Asian panels, for reasons given above (for a detailed commentary of each risk locus, see Fig. S2). With few exceptions, we also found concordance between the InORs seen in the combined Asian and combined European panels (Fig. S7).

To investigate overall concordance in the genetic basis of $\mathrm{PBC}$ between European and Asian populations, we estimated the proportion of trait variance explained (on the liability scale) in the Japanese cohort (for which individual-level genotype data were available) by sets of variants chosen according to their $p$ values in the European GWMA (see supplementary information). Regardless of the $p$ value threshold and the assumed trait prevalence, variants showing some level of association in the European GWMA explained more of the trait variance than an equivalent number of randomly chosen variants - in most instances, significantly more - supporting the conclusion that loci influencing the risk of PBC in Europeans, also influence its risk in Asians (Table S8).

Thus, while equivalently powered cohorts, accurately genotyped at the same set of variants, would be required to fully address the question of population-specific genetic heterogeneity, our results provide preliminary evidence that the genetic architecture of PBC is broadly shared across European and Asian populations.

\section{Co-localisation and DEPICT enable prioritisation of candidate genes}

In functional annotation, we found that credible causal variants included missense variants in 21 genes at 14 risk loci; splice variants in 8 genes at 5 risk loci; and stop variants in 2 genes at 2 risk loci (Table S9). Few of these variants were predicted to be deleterious. Credible causal variants at all genome-wide significant risk loci mapped to chromatin interacting regions (CIRs), mQTLs, eQTLs, or pQTLs (Tables S10-12); and in the MWAS, TWAS, and PWAS, we predicted differential methylation, transcription, or translation of genes at and beyond GWMAsignificant loci (Tables S13-15, Fig. S8). These observations suggest that the genetic architecture of PBC confers susceptibility to disease mainly by influencing the regulation of expression of causal genes. Therefore, we sought co-localisation of GWMA with mQTL, eQTL, or pQTL association signals, aiming to pinpoint causal variants and genes across the genome. Using moloc, we identified 251 co-localisation models with posterior probability of association $\geq 0.80$, implicating variants and genes at 60 loci (Table S16, Fig. S8C). Of these, 28 correspond to GWMAsignificant risk loci, where co-localisation models implicate candidate genes such as IL12RB2 (1p31.3), FCRL3 (1q23.1), and INAVA (1q32.1). Association at the other 32 loci did not reach genome-wide significance in the GWMA; co-localisation models nevertheless implicate highly plausible candidate genes at some of these loci, such as CCL21 (9p13.3) and IL2RB (22q12.3).

We found that candidate genes implicated by co-localisation were broadly concordant with those implicated by functional annotation of credible causal variants, and by the MWAS, TWAS, and PWAS. As in previous studies, we also observed that candidate genes at disparate risk loci are evidently related in function, e.g., IL12A (3q25.33), IL12B (5q33.3), IL12RB1 (19p13.11), and IL12RB2 (1p31.3). Therefore, we used DEPICT ${ }^{25}$ to prioritise candidate genes at genome-wide significant risk loci based on gene function. In this way, we identified 82 candidate genes with a false discovery rate (FDR) $<5 \%$ across 48 loci (Table S17). As expected, genes prioritised by DEPICT overlapped with those prioritised by the other approaches (Table S18).

We used the information garnered above to finalise a list of top candidate genes at genome-wide significant risk loci

Table 3. In silico drug efficacy screening.

\begin{tabular}{|c|c|c|c|}
\hline Drug name & $z$ & $p$ value & Description \\
\hline $\begin{array}{l}\text { Ustekinumab } \\
\text { Belatacept }\end{array}$ & $\begin{array}{l}-4.757 \\
-4.709\end{array}$ & $\begin{array}{l}9.82 \times 10^{-7} \\
1.24 \times 10^{-6}\end{array}$ & $\begin{array}{l}\text { Anti-IL-12/23 p40 antibody } \\
\text { IgG1 Fc/CTLA-4 fusion protein }\end{array}$ \\
\hline $\begin{array}{l}\text { Abatacept } \\
\text { Acitretin }\end{array}$ & $\begin{array}{l}-4.603 \\
-4.548\end{array}$ & $\begin{array}{l}2.08 \times 10^{-6} \\
2.71 \times 10^{-6}\end{array}$ & $\begin{array}{l}\text { IgG1 Fc/CTLA-4 fusion protein } \\
\text { Oral retinoid }\end{array}$ \\
\hline $\begin{array}{l}\text { Denosumab } \\
\text { Etretinate }\end{array}$ & $\begin{array}{l}-4.416 \\
-3.879\end{array}$ & $\begin{array}{l}5.03 \times 10^{-6} \\
5.24 \times 10^{-5}\end{array}$ & $\begin{array}{l}\text { Anti-TNFSF11 antibody } \\
\text { Oral retinoid }\end{array}$ \\
\hline $\begin{array}{l}\text { Tofacitinib } \\
\text { Basiliximab }\end{array}$ & $\begin{array}{l}-3.340 \\
-3.320\end{array}$ & $\begin{array}{l}4.19 \times 10^{-4} \\
4.50 \times 10^{-4}\end{array}$ & $\begin{array}{l}\text { Janus kinase inhibitor } \\
\text { Anti-IL2R } \alpha \text { antibody }\end{array}$ \\
\hline $\begin{array}{l}\text { Gilteritinib } \\
\text { Fostamatinib }\end{array}$ & $\begin{array}{l}-3.310 \\
-3.305\end{array}$ & $\begin{array}{l}4.66 \times 10^{-4} \\
4.75 \times 10^{-4}\end{array}$ & $\begin{array}{l}\text { Tyrosine kinase inhibitor } \\
\text { Tyrosine kinase inhibitor }\end{array}$ \\
\hline $\begin{array}{l}\text { Imatinib } \\
\text { Dexchlorpheniramine maleate }\end{array}$ & $\begin{array}{l}-3.189 \\
-3.182\end{array}$ & $\begin{array}{l}7.14 \times 10^{-4} \\
7.31 \times 10^{-4}\end{array}$ & $\begin{array}{l}\text { Tyrosine kinase inhibitor } \\
\text { Antihistamine }\end{array}$ \\
\hline $\begin{array}{l}\text { Linagliptin } \\
\text { Brigatinib }\end{array}$ & $\begin{array}{l}-3.010 \\
-2.961\end{array}$ & $\begin{array}{l}1.31 \times 10^{-3} \\
1.53 \times 10^{-3}\end{array}$ & $\begin{array}{l}\text { Dipeptidyl Peptidase-IV Inhibitor } \\
\text { ALK and EGFR inhibitor }\end{array}$ \\
\hline $\begin{array}{l}\text { Interferon alfa- } 2 \mathrm{a} \\
\text { Interferon beta-1b }\end{array}$ & $\begin{array}{l}-2.748 \\
-2.688\end{array}$ & $\begin{array}{l}3.00 \times 10^{-3} \\
3.59 \times 10^{-3}\end{array}$ & $\begin{array}{l}\text { Alpha interferon } \\
\text { Beta interferon }\end{array}$ \\
\hline $\begin{array}{l}\text { Metformin } \\
\text { Fenofibrate }\end{array}$ & $\begin{array}{l}-1.894 \\
-0.986\end{array}$ & $\begin{array}{l}0.029 \\
0.162\end{array}$ & $\begin{array}{l}\text { Biguanide antidiabetic agent } \\
\text { Fibrate, PPAR- } \alpha \text { agonist }\end{array}$ \\
\hline $\begin{array}{l}\text { Bezafibrate } \\
\text { Obeticholic acid }\end{array}$ & $\begin{array}{l}-0.866 \\
-0.737\end{array}$ & $\begin{array}{l}0.193 \\
0.231\end{array}$ & $\begin{array}{l}\text { Fibrate, PPAR- } \alpha / \delta / \gamma \text { agonist } \\
\text { Bile acid, FXR agonist }\end{array}$ \\
\hline Rifampicin & -0.627 & 0.265 & Antibiotic \\
\hline Ursodeoxycholic acid & +0.171 & 0.568 & Bile acid \\
\hline
\end{tabular}

Results for top-ranking agents and current treatments for primary biliary cholangitis, $z$ being a drug-disease proximity measure, defined as $z=\left(d_{c}-\mu\right) / \sigma$ where $d_{c}$ is the average shortest path length between the drug's targets and the nearest disease gene, and $\mu$ and $\sigma$ are calculated via a randomisation procedure as described in the supplementary information. Guney et al. define a drug to be proximal to a disease if its proximity follows $z \leq-0.15$ ( $p \leq 0.44$ ), and distant otherwise. 
(Table S18). Using STRING, ${ }^{26}$ we found these genes to be highly enriched for protein-protein interactions $\left(p<1.0 \times 10^{-16}\right)$, with enrichment at FDR $<5 \%$ of the following KEGG pathways: $T$ helper $\left(T_{H}\right) 1$ and $T_{H} 2$ cell differentiation, $T_{H} 17$ cell differentiation, and toll-like receptor (TLR), RIG-I-like receptor (RLR), TNF, NF- $\kappa B$, and JAK-STAT signalling pathways, amongst others (Fig. S9). For comparison, we undertook enrichment analysis using DAVID ${ }^{27}$ of 1,388 genes with minimum $p_{\mathrm{GWMA}}<0.01$, which identified enrichment at FDR $<5 \%$ of the following KEGG pathways: antigen processing and presentation, Fc $\gamma$ R-mediated phagocytosis, NK cell-mediated cytotoxicity, and T cell receptor, B cell receptor, PI3K-AKT, FceRI, JAK-STAT, NF- $\kappa B$, and MAPK signalling pathways, amongst others (Table S19).

\section{In silico drug efficacy screening identifies agents potentially suitable for re-purposing to PBC}

In the approach of Guney et al. (2016), ${ }^{28}$ the more negative the value of $z$, the closer the drug and disease gene networks. A cutoff of $z \leq-0.15$ is taken to show that the drug is proximal to the disease and thus, might exert pharmacological effects on it. In our analysis, we identified many agents with $z \leq-0.15$, which are therefore predicted to exert pharmacological effects on PBC (Table 3, Table S20). Top-ranking drugs that might be predicted to ameliorate PBC included several immunomodulators, such as ustekinumab, an anti-IL-12/23 monoclonal antibody used for psoriasis and Crohn's disease $(z=-4.757)$; belatacept, a CTLA-4 fusion protein used in organ transplantation $(z=-4.709)$; and abatacept, a CTLA-4 fusion protein used for RA, juvenile idiopathic arthritis (JIA), and psoriatic arthritis $(z=-4.603)$. Of interest, other top-ranking agents include the retinoids etretinate and its metabolite acitretin, both of which are used for the treatment of psoriasis ( $z=-3.879$ and $z=-4.548$, respectively). Top-ranking drugs that might be predicted to exacerbate PBC included the pharmacological interferons, such as interferon alfa-2a and interferon beta- $1 \mathrm{~b}(z=-2.748$ and $z=-2.688$, respectively). Amongst recognised treatments for $\mathrm{PBC}$, fenofibrate scored $z=-0.986$; bezafibrate, $z=-0.866$; and OCA, $z=$ -0.737 , respectively. Thus, these drugs might be predicted to exert pharmacological effects on PBC. Conversely, UDCA scored $z=+0.171$, meaning it is not predicted to treat the genetically determined component of disease in PBC.

\section{Discussion}

We report the largest GWMA of PBC undertaken to date, with a sample size four times greater than that of our previous study. In this better-powered study, we identified 21 additional genomewide significant risk loci; showed that the genetic architecture of PBC is broadly shared across European and Asian populations; prioritised candidate genes at known and newly identified genome-wide significant risk loci; and used these candidate genes to identify medications predicted to treat the genetically determined component of disease in $\mathrm{PBC}$, which might therefore be suitable for re-purposing to this condition.

Candidate genes at newly identified or newly confirmed risk loci provide additional insights into the pathogenesis of $\mathrm{PBC}$ (Fig. 1). Thus, INAVA (1q32.1) amplifies pattern recognition receptor (PRR) signalling; DNMT3A (2p23.3), ZC3HAV1 (7q34), and TRIM14 (9q22.33) are each involved in RLR signalling; TET2 (4q24) represses transcription of IL-6; and PVT1 (8q24.21) regulates inflammation via NF- $\kappa \mathrm{B}$ and MAPK pathways. Chemokine receptor $6(C C R 6,6 q 27)$ interacts with CCL20 in the chemotaxis of dendritic cells and lymphocytes to inflamed epithelia; ST8SIA4 (5q21.1) is required for the interaction of CCR7 with CCL21 in the trafficking of immune cells to secondary lymphatic organs; and CD226 (18q22.2) participates in lymphocyte and NK cell adhesion and signalling. Fc receptor-like protein 3 (FCRL3, 1q23.1), ID2 (2p25.1), TET2 (4q24), RARB (3p24.2), NDFIP1 (5q31.3), ITGB8 (7p21.1), and $C D 226(18 q 22.2)$ are each involved in the differentiation of $T_{H} 1, T_{H} 17$, or regulatory $T$ cells. As expected, enrichment analysis of candidate genes reiterated the importance of PRR, TNF, and NF- $\kappa B$ signalling, and $T_{H} 1 / T_{H} 17$ cell differentiation in this disease. These findings are consistent with functional data emphasising the importance of innate immune cell hypersensitivity, chemokine signalling and immune cell trafficking, and $\mathrm{T}_{\mathrm{H}} 1 / \mathrm{T}_{\mathrm{H}} 17$ cell polarisation in $\mathrm{PBC}$ pathogenesis, as summarised by Gulamhusein and Hirschfield $(2020)^{31}$ in their recent review.

There is considerable current interest in the 'Druggable Genome', i.e., the use of genome-wide approaches to find targets for drug discovery (for example, see the Open Targets initiative at https://www.opentargets.org/). In the current study, having prioritised candidate genes, we used network-based in silico drug efficacy screening to identify agents potentially suitable for repurposing to PBC. Given our other findings - including genetic correlation of PBC with SLE, RA, and IBD - it is expected that the top-ranking medications should include immunomodulators already approved for the treatment of RA, JIA, IBD, MS, or psoriasis.

The evidence to support re-purposing of these immunomodulators to PBC is circumstantial yet convincing - but circumspection is required. For example, in the current study, LDSR demonstrated genetic correlation with IBD; enrichment analysis showed association with ' $\mathrm{T}_{\mathrm{H}} 1$ and $\mathrm{T}_{\mathrm{H}} 2$ cell differentiation'; and drug efficacy screening suggested that ustekinumab, an anti-IL-12/23 monoclonal antibody used for treatment of Crohn's disease, might exert pharmacological effects on PBC. Therefore, it is notable that ustekinumab showed minimal effect on PBC in the trial by Hirschfield et al. (2016). ${ }^{32}$ Similarly, drug efficacy screening suggested that abatacept, a CTLA-4 fusion protein used for treatment of RA, might be effective for treatment of PBC - but abatacept showed no effect on PBC in the trial by Bowlus et al. (2019). ${ }^{33}$ A potential explanation for these discrepant observations, also expounded by Bowlus et al., ${ }^{33}$ is that the evaluation of immunomodulators in $\mathrm{PBC}$ might require a change in clinical trial design. Thus, immunomodulators might require immunological rather than cholestatic endpoints; might be more effective in early disease, before the cholestatic liver injury predominates; and might require combined treatment of both the autoimmune and cholestatic injuries. Re-design of clinical trials in PBC might be contentious but the use of genomic data to prioritise potential agents for $\mathrm{PBC}$ is not, as new treatments for $\mathrm{PBC}$ are needed and the druggable genome provides a framework to find them.

It is notable that in drug efficacy screening, UDCA - wellestablished as first-line treatment for PBC - was not predicted to be therapeutic in this condition. One possibility is that UDCA serves primarily to treat a cholestatic liver injury that is critical to disease progression but orthogonal to the genetically determined, autoimmune processes that confer risk of disease. Conversely, OCA (a potent FXR agonist) and the fibrates, bezafibrate and fenofibrate (PPAR- $\alpha / \delta / \gamma$ and PPAR- $\alpha$ agonists, 


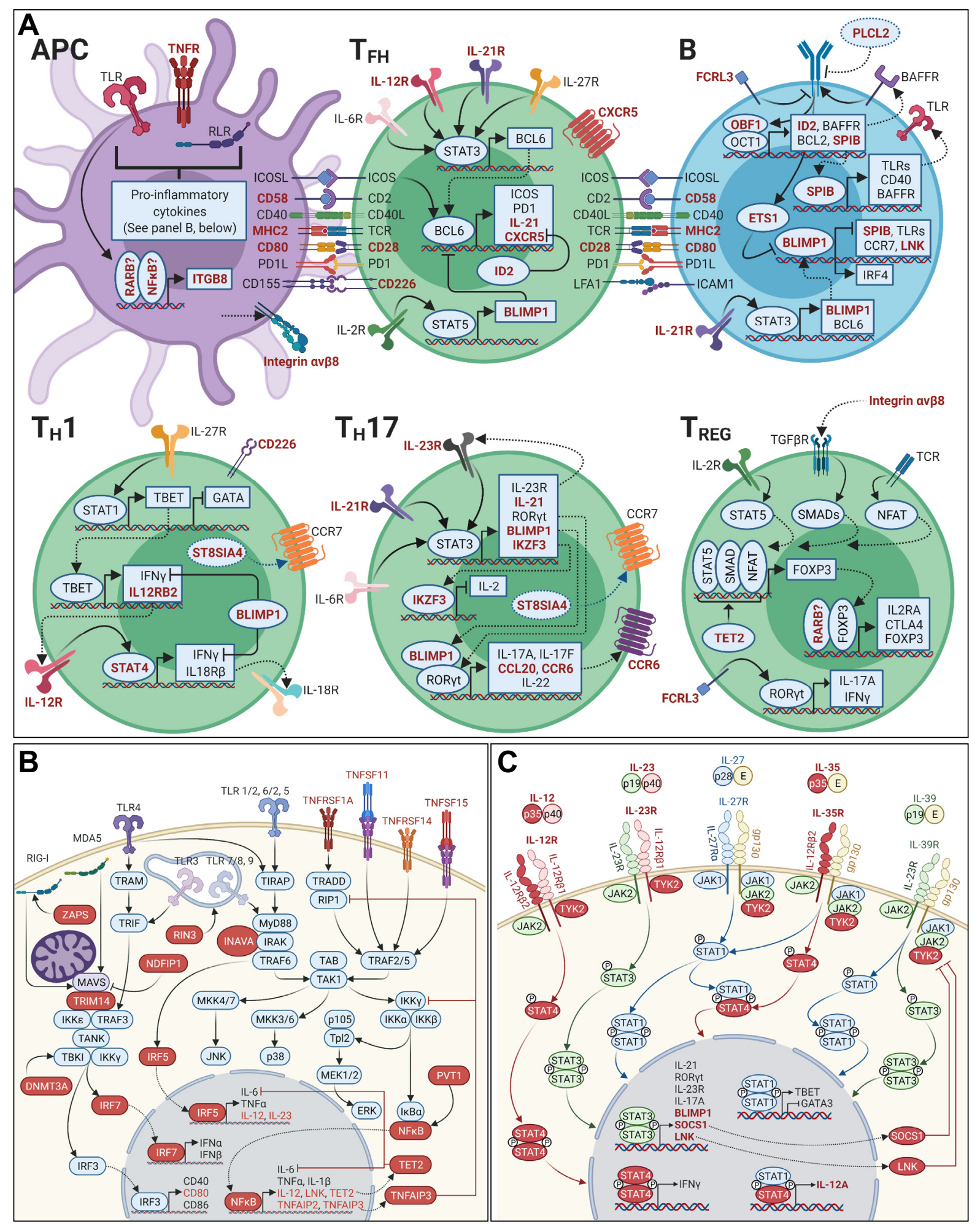

Fig. 1. Biological processes implicated by candidate genes (red bold or red filled) prioritised in the current study. (A) $\mathrm{T}$ and $\mathrm{B}$ cell activation, and differentiation of $\mathrm{T}$ follicular helper, $\mathrm{T}_{\mathrm{H}} 1, \mathrm{~T}_{\mathrm{H}} 17$, and $\mathrm{T}_{\mathrm{REG}}$ cells; (B) pattern recognition receptor and TNF signalling in antigen presenting cells; and (C) signalling by the IL-12 family of cytokines. $\mathrm{T}_{\mathrm{H}}$, $\mathrm{T}$ helper; $\mathrm{T}_{\mathrm{REG}}$, regulatory $\mathrm{T}$. (Figure created with BioRender.com). 
respectively), are expected to have immune-modulatory as well as anti-cholestatic effects. ${ }^{34,35}$

We acknowledge 2 major limitations of the study. First, the absence of an independent validation cohort meant we were unable to confirm several newly identified risk loci. Other strategies, such as cross-phenotype meta-analysis, may be required for external validation of these loci. Second, the use of different genotyping platforms across cohorts meant that at many risk loci, the lead variant in the European panels was not represented in the Asian panels, or vice versa. This, together with marked disparity in the sample size of the European vs. the Asian panels, meant that we were unable to fully address the question of population-specific genetic heterogeneity.

In conclusion, our large, trans-ethnic GWMA of PBC has identified additional risk loci; found little evidence for population-specific genetic heterogeneity; and, through functional annotation of credible causal variants and multi-omic analysis, allowed us to prioritise candidate genes, and thereby prioritise drugs potentially suitable for re-purposing to $\mathrm{PBC}$. This study emphasises the value of genomic approaches to provide biological insight and guide the development of novel therapies.

\section{Abbreviations}

ALSPAC, Avon Longitudinal Study of Parents and Children; COJO, Conditional and Joint Analysis; eQTL, expression quantitative trait locus; FDR, false discovery rate; FUMA, functional mapping and annotation; GC, genomic control; GTEx, genotype-tissue expression; GWAS, genome-wide association study; GWMA, genome-wide meta-analysis; HLA, human leukocyte antigen; HWE, Hardy-Weinburg equilibrium; IBD, inflammatory bowel disease; JIA, juvenile inflammatory arthritis; LD, linkage disequilibrium; LDSR, linkage disequilibrium score regression; MAF, minor allele frequency; mQTL, methylation quantitative trait locus; MS, multiple sclerosis; MWAS, methylome-wide association study; OCA, obeticholic acid; OR, odds ratio; PBC, primary biliary cholangitis; pQTL, protein-quantitative trait locus; PWAS, proteome-wide association study; QC, quality control; RA, rheumatoid arthritis; SLE, systemic lupus erythematosus; SNP, single nucleotide polymorphism; $\mathrm{T}_{\mathrm{H}}, \mathrm{T}$ helper; TWAS, transcriptome-wide association study; UDCA, ursodeoxycholic acid.

\section{Financial support}

HJC is funded by a Wellcome Trust Senior Research Fellowship in Basic Biomedical Science (102858/Z/13/Z). JJF is funded by a BBSRC DTP studentship (BB/M011186/1). The University of Cambridge has received salary support in respect of RNS from the NHS in the East of England through the Clinical Academic Reserve. KNL was supported by the NIH, DK80670. CIA is a Cancer Prevention Research Institute of Texas (CPRIT) Established Scholar and is supported by RR170048 and CA186566. AG, MC, and PI are supported by unrestricted research funding from AMAF Monza ONLUS and AIRCS, and partially supported by the Italian Ministry of University and Research (MIUR) - Department of Excellence project PREMIA (PREcision MedIcine Approach: bringing biomarker research to clinic). KAS is supported by the Sherman Family Chair in Genomic Medicine and a Foundation grant from the Canadian Institutes for Health Research (353710) and an Ontario Research Fund award (RE-09090). MN is funded by a Grant-in-Aid for Clinical Research from the National Hospital Organization and grants from Japan Society for the
Promotion of Science (26293181, 17H04169). KT and MN are funded by grants from Japan Agency for Medical Research and Development (AMED) (JP20km0405205 and JP20km0405501). GFM was funded by a post-doctoral fellowship from the National Institute for Health Research (NIHR) Rare Diseases - Translational Research Collaboration (RD-TRC) and is now funded by a Clinician Academic Research Partnership (CARP) award from the Medical Research Council (MRC), UK. The MRC, UK (Grant ref: 217065/Z/19/Z) and the University of Bristol provide core support for ALSPAC, and the ALSPAC GWAS data were generated by Sample Logistics and Genotyping Facilities at Wellcome Sanger Institute and LabCorp (Laboratory Corporation of America) using support from 23andMe. A comprehensive list of grants funding is available on the ALSPAC website (http://www.bristol.ac.uk/ alspac/external/documents/grant-acknowledgements.pdf). The research included in the current study involved collection of genotype data from the ARIES mothers funded by the Wellcome Trust (WT088806), and collection of the ARIES methylation data funded by the BBSRC (BBI025751/1 and BB/I025263/1). UK-PBC was funded by a Stratified Medicine award from the MRC, UK (MR/L001489/1).

\section{Conflicts of interest}

GMH has consulted and/or been a speaker for Intercept, Genfit, Cymabay, GSK, and Falk. RNS and GFM have each received research funding from Intercept Pharmaceuticals. $\mathrm{HJC}$, JJF, KU, RD, YA, YH, MK, NN, S-SK, OG, YK, MN, KT, RT, YS, ZL, BDJ, EJA, AG, MC, RA, AC, MdA, AB, JH, MARF, DS, DEJ, SF, AS, VLM, KNL, CIA, MFS, PI, KAS, XM and MN report no conflicts of interest.

Please refer to the accompanying ICMJE disclosure forms for further details.

\section{Authors' contributions}

HJC, DEJ, RNS, KNL, PI, MFS, KAS, CIA, XM, MN and GFM conceived and planned the project. KNL, PI, MFS, KAS, XM, MN and GFM directed recruitment, sample collection and genotyping. $\mathrm{HJC}, \mathrm{JJF}, \mathrm{KU}, \mathrm{RD}$ and CIA carried out the analyses. All authors contributed to interpretation of the results. HJC and GFM wrote the manuscript. All authors provided critical feedback and shaped the analysis and manuscript.

\section{Data availability statement}

Following publication, summary statistics from the current study will be deposited with the European Genome-phenome Archive.

\section{Acknowledgements}

We are grateful to Dr Carl Anderson at the Wellcome Sanger Institute for facilitating genotyping of the UK-PBC Research Cohort. AG, MC, and PI are members of the European Reference Network on Hepatological Diseases (ERN RARE-LIVER). We are grateful to all the families who took part in ALSPAC, the midwives for their help in recruiting them, and the whole ALSPAC team, which includes interviewers, computer and laboratory technicians, clerical workers, research scientists, volunteers, managers, receptionists, and nurses. We are grateful to all the people with $\mathrm{PBC}$ enrolled in the respective $\mathrm{PBC}$ resource cohorts included in the current study. 


\section{JOURNAL OF HEPATOLOGY}

\section{Supplementary data \\ Supplementary data to this article can be found online at https:// doi.org/10.1016/j.jhep.2021.04.055.}

\section{References}

[1] Hirschfield GM, Liu X, Xu C, Lu Y, Xie G, Lu Y, et al. Primary biliary cirrhosis associated with HLA, IL12A, and IL12RB2 variants. N Engl J Med 2009;360:2544-2555.

[2] Liu X, Invernizzi P, Lu Y, Kosoy R, Lu Y, Bianchi I, et al. Genome-wide metaanalyses identify three loci associated with primary biliary cirrhosis. Nat Genet 2010;42:658-660.

[3] Mells GF, Floyd JA, Morley KI, Cordell HJ, Franklin CS, Shin SY, et al. Genome-wide association study identifies 12 new susceptibility loci for primary biliary cirrhosis. Nat Genet 2011;43:329-332.

[4] Kawashima M, Hitomi Y, Aiba Y, Nishida N, Kojima K, Kawai Y, et al Genome-wide association studies identify PRKCB as a novel genetic susceptibility locus for primary biliary cholangitis in the Japanese population. Hum Mol Genet 2017;26:650-659.

[5] Qiu F, Tang R, Zuo X, Shi X, Wei Y, Zheng X, et al. A genome-wide association study identifies six novel risk loci for primary biliary cholangitis. Nat Commun 2017;8:14828.

[6] Cordell HJ, Han Y, Mells GF, Li Y, Hirschfield GM, Greene CS, et al. International genome-wide meta-analysis identifies new primary biliary cirrhosis risk loci and targetable pathogenic pathways. Nat Commun 2015;6:8019.

[7] Purcell S, Neale B, Todd-Brown K, Thomas L, Ferreira MA, Bender D, et al PLINK: a tool set for whole-genome association and population-based linkage analyses. Am J Hum Genet 2007;81:559-575.

[8] Loh PR, Danecek P, Palamara PF, Fuchsberger C, Reshef YA, Finucane HK et al. Reference-based phasing using the haplotype reference consortium panel. Nat Genet 2016;48:1443-1448.

[9] Delaneau O, Marchini J, Zagury JF. A linear complexity phasing method for thousands of genomes. Nat Methods 2011:9:179-181.

[10] Howie BN, Donnelly P, Marchini J. A flexible and accurate genotype imputation method for the next generation of genome-wide association studies. Plos Genet 2009;5:e1000529.

[11] Chen H, Wang C, Conomos MP, Stilp AM, Li Z, Sofer T, et al. Contro for population structure and relatedness for binary traits in genetic association studies via logistic mixed models. Am J Hum Genet 2016;98:653-666.

[12] Liu JZ, Tozzi F, Waterworth DM, Pillai SG, Muglia P, Middleton L, et al. Meta-analysis and imputation refines the association of $15 q 25$ with smoking quantity. Nat Genet 2010;42:436-440.

[13] Bulik-Sullivan BK, Loh PR, Finucane HK, Ripke S, Yang J, Schizophrenia Working Group of the Psychiatric Genomics C, et al. LD Score regression distinguishes confounding from polygenicity in genome-wide association studies. Nat Genet 2015;47:291-295.

[14] Magi R, Horikoshi M, Sofer T, Mahajan A, Kitajima H, Franceschini N, et al. Trans-ethnic meta-regression of genome-wide association studies accounting for ancestry increases power for discovery and improves finemapping resolution. Hum Mol Genet 2017;26:3639-3650.

[15] Benner C, Spencer CC, Havulinna AS, Salomaa V, Ripatti S, Pirinen M. FINEMAP: efficient variable selection using summary data from genomewide association studies. Bioinformatics 2016;32:1493-1501.

[16] Yang J, Ferreira T, Morris AP, Medland SE, Genetic Investigation of ATC, Replication DIG, et al. Conditional and joint multiple-SNP analysis of GWAS summary statistics identifies additional variants influencing complex traits. Nat Genet 2012;44:369-375. S361-363.
[17] Yang J, Lee SH, Goddard ME, Visscher PM. GCTA: a tool for genome-wide complex trait analysis. Am J Hum Genet 2011;88:76-82.

[18] McLaren W, Gil L, Hunt SE, Riat HS, Ritchie GR, Thormann A, et al. The ensembl variant effect predictor. Genome Biol 2016;17:122.

[19] Watanabe K, Taskesen E, van Bochoven A, Posthuma D. Functional mapping and annotation of genetic associations with FUMA. Nat Commun 2017;8:1826

[20] Relton CL, Gaunt T, McArdle W, Ho K, Duggirala A, Shihab H, et al. Data resource profile: accessible resource for integrated epigenomic studies (ARIES). Int J Epidemiol 2015;44:1181-1190.

[21] Sun BB, Maranville JC, Peters JE, Stacey D, Staley JR, Blackshaw J, et al, Genomic atlas of the human plasma proteome. Nature 2018;558:73-79.

[22] Barbeira AN, Dickinson SP, Bonazzola R, Zheng J, Wheeler HE, Torres JM, et al. Exploring the phenotypic consequences of tissue specific gene expression variation inferred from GWAS summary statistics. Nat Commun 2018;9:1825.

[23] Consortium GT. The genotype-tissue expression (GTEx) project. Nat Genet 2013;45:580-585.

[24] Giambartolomei C, Zhenli Liu J, Zhang W, Hauberg M, Shi H, Boocock J, et al. A Bayesian framework for multiple trait colocalization from summary association statistics. Bioinformatics 2018;34:2538-2545.

[25] Pers TH, Karjalainen JM, Chan Y, Westra HJ, Wood AR, Yang J, et al. Biological interpretation of genome-wide association studies using predicted gene functions. Nat Commun 2015;6:5890.

[26] Szklarczyk D, Gable AL, Lyon D, Junge A, Wyder S, Huerta-Cepas J, et al. STRING v11: protein-protein association networks with increased coverage, supporting functional discovery in genome-wide experimental datasets. Nucleic Acids Res 2019;47:D607-D613.

[27] Huang da W, Sherman BT, Lempicki RA. Bioinformatics enrichment tools: paths toward the comprehensive functional analysis of large gene lists. Nucleic Acids Res 2009;37:1-13.

[28] Guney E, Menche J, Vidal M, Barabasi AL. Network-based in silico drug efficacy screening. Nat Commun 2016;7:10331.

[29] Tanaka A, Ohira H, Kikuchi K, Nezu S, Shibuya A, Bianchi I, et al. Genetic association of Fc receptor-like 3 polymorphisms with susceptibility to primary biliary cirrhosis: ethnic comparative study in Japanese and Italian patients. Tissue Antigens 2011;77:239-243.

[30] Zheng J, Erzurumluoglu AM, Elsworth BL, Kemp JP, Howe L, Haycock PC, et al. LD Hub: a centralized database and web interface to perform LD score regression that maximizes the potential of summary level GWAS data for SNP heritability and genetic correlation analysis. Bioinformatics 2017:33:272-279.

[31] Gulamhusein AF, Hirschfield GM. Primary biliary cholangitis: pathogenesis and therapeutic opportunities. Nat Rev Gastroenterol Hepatol 2020;17:93-110.

[32] Hirschfield GM, Gershwin ME, Strauss R, Mayo MJ, Levy C, Zou B, et al. Ustekinumab for patients with primary biliary cholangitis who have an inadequate response to ursodeoxycholic acid: a proof-of-concept study. Hepatology 2016;64:189-199.

[33] Bowlus CL, Yang GX, Liu CH, Johnson CR, Dhaliwal SS, Frank D, et al. Therapeutic trials of biologics in primary biliary cholangitis: an open label study of abatacept and review of the literature. J Autoimmun 2019:101:26-34.

[34] Chen ML, Takeda K, Sundrud MS. Emerging roles of bile acids in mucosal immunity and inflammation. Mucosal Immunol 2019;12:851-861.

[35] Christofides A, Konstantinidou E, Jani C, Boussiotis VA. The role of peroxisome proliferator-activated receptors (PPAR) in immune responses. Metabolism 2021;114:154338. 